\title{
Context specificity of conditioning in flavor-aversion learning: Extinction and blocking tests
}

\author{
CHARLOTTE BONARDI, R. C. HONEY, and GEOFFREY HALL \\ University of York, York, England
}

\begin{abstract}
In three experiments using rats as subjects, we investigated the degree to which a conditioned flavor aversion transfers from one context to another. Experiment 1, using a one-trial conditioning procedure, found no effect of a change of context on a conditioned aversion. Experiment 2 employed a multitrial procedure and demonstrated that a conditioned aversion was extinguished more rapidly after a change of context. Experiment 3 showed that context change decreased the effectiveness with which a conditioned flavor could block acquisition of an aversion by a second flavor. It is argued that these data cannot be explained in associative terms, and that they constitute evidence of conditionality in a simple aversive conditioning procedure.
\end{abstract}

It is well established that explicit discrimination training can render the effects of simple conditioning contextdependent. Thus, an animal given pairings of a conditioned stimulus (CS) and an unconditioned stimulus (US) in one distinctive context, and presentations of the same $\mathrm{CS}$ along with some other outcome in a different context, will come to emit different conditioned responses (CRs) in the two contexts (e.g., see Bouton \& Swartzentruber, 1986; Preston, Dickinson, \& Mackintosh, 1986). Such context dependency has been interpreted as lying beyond the scope of associative theory and as showing that contextual cues can come to modulate CS-US associations, providing information that a specific CS-US relationship is in force, or helping the subject retrieve information about the consequences of the CS.

There is considerable room for doubt, however, that context dependency of this sort can be established without explicit discrimination training. If the animal is exposed to CS-US pairings only in a given context, but is not exposed to the CS elsewhere-as occurs in a simple conditioning procedure-will the contextual cues nonetheless acquire conditional control over the ability of the CS to evoke the CR?

The simplest test of this idea is to conduct conditioning in one distinctive context and then to examine the ability of the CS to evoke the CR in some other context. In some experiments using this procedure, a loss of conditioned responding has been observed (although this outcome is not as common as is often supposed; see Bouton, 1988, for a review of this issue). But the failure of the CR to transfer across contexts does not necessarily mean that the context has acquired some higher level ability to modu-

This research was financed by grants from the Science and Engineering Research Council. Correspondence regarding this paper may be addressed to Charlotte Bonardi, University of York, Heslington, York YO1 5DD, England. late the operation of the CS-US association. As Lovibond, Preston, and Mackintosh (1984) have pointed out, alternative, simpler explanations are available. One possibility is that the change of context modifies the way in which the CS is perceived, thus producing generalization decrement. The second possibility is that the magnitude of the CR depends on a contribution from a context-US association that will be lacking when the CS is presented in a context other than that used for training. In experiments using the conditioned suppression procedure that were designed to eliminate these confounding factors, Lovibond et al. were able to demonstrate perfect transfer of the CR across contexts. In only one set of studies (by Hall \& Honey, 1989) has context specificity of conditioning been seen when the experimental design has incorporated the controls urged by Lovibond et al.

A feature of the experiments reported by Hall and Honey (1989) was that an appetitive reinforcer was used, a feature that appears to be critical in that Hall and Honey also report a further study using the conditioned suppression procedure in which (as in the experiments of Lovibond et al., 1984) no context specificity was evident. The appetitive results may be enough in themselves to demonstrate that simple conditioning can in some circumstances cause contextual cues to acquire control over a CS-US association; nonetheless, the failure to find an effect in conditioned suppression requires explanation. Hall and Honey put forward several possible explanations, one of which was based on the suggestion that aversive reinforcement disrupts the processes required for encoding information about conditioned stimuli. An implication of this suggestion, that context specificity should not be found whenever a potent aversive US is employed, forms the focus of the present paper.

The experiments we describe represent an attempt to demonstrate that a conditioned aversion to a flavor will transfer only poorly to a context other than that used for 
training, and that the mechanism responsible for this context specificity appears to require us to acknowledge that the context exerts conditional control over the CS-US association, despite the use of an aversive reinforcer. We chose the flavor-aversion preparation for two reasons. First, previous work using the same general procedures as those used in the present experiments has shown that the latent inhibition produced by preexposure to a flavor will fail to transfer to a new context (Hall \& Channell, 1986). Evidently our subjects can discriminate between the contexts we use, and this discrimination can influence their response to flavors. Second, although it cannot be eliminated entirely, the scope for explaining any loss of conditioned responding in terms of generalization decrement is reduced when a flavor constitutes the critical stimulus. It seems quite likely that a tone or a light could be perceived differently according to the place in which it is encountered, but it seems somewhat less probable that the perception of a flavor would be substantially altered by the primarily exteroceptive cues that constitute the context, provided that care is taken to present the flavored substance in an identical fashion in each context.

\section{EXPERIMENT 1}

A series of experiments by Archer and his collaborators (e.g., Archer \& Sjödén, 1980; Archer, Sjödén, Nilsson, \& Carter, 1979, 1980) has convincingly demonstrated that the aversion conditioned to a saccharin solution presented to rats in one context will not transfer fully to saccharin presented in another context. However, this result cannot be taken as a demonstration of conditional control, nor indeed was it intended to do so. Rather, it was interpreted as evidence that associations involving contextual cues (e.g., with the US and with other aspects of the conditioning situation) contributed to the suppression of consumption shown after conditioning (Archer, Sjödén, \& Nilsson, 1985). In the present experiment, we looked for an effect over and above anything that might be produced by summation of CS-US and context-US associations, adopting the control procedure used by Lovibond et al. (1984). Subjects received initial conditioning with the target flavor in the training context and conditioning with some other, quite different flavor in the context that was to be used in the test. The two sets of contextual cues should therefore acquire equal associative strength so that a loss of the CR to the target flavor on test could not be the result of removing the contribution of a context-US association.

Having argued above that the perception of a given flavor is unlikely to be changed by the context in which it is presented, a qualification should be noted. Archer and his associates used different bottles to present the different flavors in their different contexts; indeed, they suggested that the type of spout used to deliver the fluid was the most important aspect of each context. It has been suggested, however (Holder, 1988; but see also Sjödén \& Archer, 1988), that the two types of bottle might have generated discriminably different tastes. To avoid any such possibility, we used identical bottles and spouts in our various contexts.

In Experiment 1, all subjects received a single conditioning trial with each of two flavors. A target flavor (T) was paired with illness in one distinctive context (a cage of a particular sort) and a control flavor (C) was paired with illness in a different context. The subjects were then divided into two groups. Group $S$ (same) was tested with $T$ in the conditioning context; Group D (different) received $T$ in the other context. Context specificity of flavor-aversion learning would be revealed by greater consumption on the test by Group D.

\section{Method}

Subjects and Apparatus. The subjects were 16 male Wistar rats with a mean free-feeding weight of $374 \mathrm{~g}$ (range: $315-450 \mathrm{~g}$ ). They were housed in pairs in cages of opaque plastic $(38 \mathrm{~cm}$ long, $26 \mathrm{~cm}$ wide, and $19 \mathrm{~cm}$ high), with wood shavings as bedding. Food was always freely available in these cages. Tap water was supplied from bottles with stainless steel, ball-bearing-tipped spouts. These cages were kept in the colony room, which was brightly lit from 0800 to $2000 \mathrm{~h}$ each day.

One training context (A) was provided by 16 cages $(50 \mathrm{~cm}$ long, $32 \mathrm{~cm}$ wide, and $19 \mathrm{~cm}$ high) of opaque plastic that were kept in the colony room. The floors of these cages were bare. Sessions in this context always began at $1100 \mathrm{~h}$ and lasted for $1 \mathrm{~h}$.

The second context (B) was provided by 16 cages $(26 \mathrm{~cm}$ long, $12 \mathrm{~cm}$ wide, and $19 \mathrm{~cm}$ high) made of transparent plastic, with wood shavings as bedding. These were kept in a small room separate from the colony room. Illumination was provided by a single $100-\mathrm{W}$ desk lamp; continuous white noise was present, with an intensity of $80 \mathrm{~dB}$ measured at the cage nearest to the loudspeaker. Sessions in this context always began at $1600 \mathrm{~h}$ and lasted for $1 \mathrm{~h}$.

Procedure. A schedule of water deprivation was established during the 7 days before the start of the experiment proper. The water bottles were removed at $1800 \mathrm{~h}$ on the first day of water deprivation, and on each subsequent day they were presented for a fixed period of time, which always ended at $1800 \mathrm{~h}$. This time period was $4 \mathrm{~h}$ on the first day, $2 \mathrm{~h}$ on the second, $1 \mathrm{~h}$ on the third, and $30 \mathrm{~min}$ on each of the last 3 days.

From this point, the animals received access to fluid only in the experimental contexts. There were two context sessions on each of the following 9 days, one in the A context and one in the B context. During these sessions, fluid was delivered from calibrated 50-ml centrifuge tubes, equipped with the same drinking spouts as the water bottles. The amount consumed was recorded to the nearest $0.5 \mathrm{ml}$. All context sessions followed the same pattern. The subjects were placed in the context and left undisturbed for $20 \mathrm{~min}$ before the tubes were presented. After $15 \mathrm{~min}$, the tubes were removed, and after a further $25 \mathrm{~min}$ the animals were returned to their home cages.

On Day 1, $10 \mathrm{ml}$ of water was presented in each session, so that the animals would become accustomed to drinking in the contexts. Conditioning with each of the two flavored solutions, $0.01 \mathrm{M} \mathrm{HCl}$ and $0.16 \mathrm{M} \mathrm{NaCl}$, occurred on Days 2 and 3. All animals received one conditioning trial on each day. During these trials, $10 \mathrm{ml}$ of one of the flavors was presented, and this was followed by a $10-\mathrm{ml} / \mathrm{kg}$ injection of $0.3 \mathrm{M} \mathrm{LiCl}$ administered after the animals had been returned to their home cages. Water was presented during the session in the other context. For half the animals, the target flavor, $\mathrm{T}$, was $\mathrm{NaCl}$, and for the remainder it was $\mathrm{HCl}$. For half of each of these two subgroups, Flavor $T$ was presented in Context $A$, and for the remainder it was presented in Context $B$. For half of each of these four subgroups, Flavor $T$ was conditioned on 
Day 2, and for the remainder it was conditioned on Day 3; there were thus eight subgroups in total.

There followed six test sessions. The subjects were divided into two groups, each consisting of 1 animal from each of the eight subgroups described above. For Group $S$ (same), the test context was that in which the target flavor had been conditioned; for Group D (different), it was the alternative context. The subjects continued to receive morning and afternoon context sessions as before. In the test context, the subjects received free access to the target solution; in the alternative context, $10 \mathrm{ml}$ of water was presented.

\section{Results and Discussion}

In all the experiments reported, inspection of the data revealed that the animals tended to drink less $\mathrm{HCl}$ than $\mathrm{NaCl}$, but that the identity of the target flavor did not alter the pattern of the test results. Moreover, there was no discernible effect of the order of conditioning upon fluid consumption in the test. These factors were therefore not included in the statistical analyses. There was, however, a consistent tendency for the subjects to consume more fluid in Context A than in Context B, and so the identity of the test context was included as a factor in the analysis of the test data.

The subjects drank similar amounts of Flavors $\mathrm{T}$ and $\mathrm{C}$ on the single conditioning trial. Mean consumption of Flavor $\mathrm{T}$ was $7.63 \mathrm{ml}$ for Group $\mathrm{S}$ and $7.50 \mathrm{ml}$ for Group D; the corresponding means for Flavor $\mathrm{C}$ were 7.31 and $6.94 \mathrm{ml}$. An analysis of variance (ANOVA) with group ( $\mathrm{S}$ or $\mathrm{D}$ ) and flavor ( $\mathrm{T}$ or $\mathrm{C}$ ) as factors revealed no significant effects or interactions $(F \mathrm{~s}<1)$.

Figure 1 shows mean fluid consumption during the test phase. It is clear that the conditioning procedure successfully established an aversion to Flavor $T$; both groups showed a marked initial suppression of consumption that diminished with repeated testing. There was no very clear difference between the groups, however. Group S showed somewhat less consumption on the first three test sessions, and somewhat more on the later sessions, but these differences proved to be statistically unreliable. An ANOVA with group (S or D), test context (A or B), and sessions (three-session blocks) as factors revealed a main effect of sessions $[F(1,12)=20.75, p<.01]$, but there was no main effect of group $(F<1)$, and the group $\times$ sessions interaction fell short of significance $[F(1,12)=$ 4.42]. No other effects or interactions were significant [largest $F(1,12)=1.60$ ].

Our failure to demonstrate context specificity of conditioning in this experiment suggests that the differences between the procedure used here and those used by Archer et al. $(1979,1980)$ were successful in eliminating the source of the effect seen by Archer et al. Our procedure was intended to exclude generalization decrement as much as possible, and to equate for the two groups the strength of any direct association between contextual cues and the US. These factors may well have played a role in the context specificity demonstrated in the earlier experiments. Archer et al. (1985), in fact, interpreted their result as

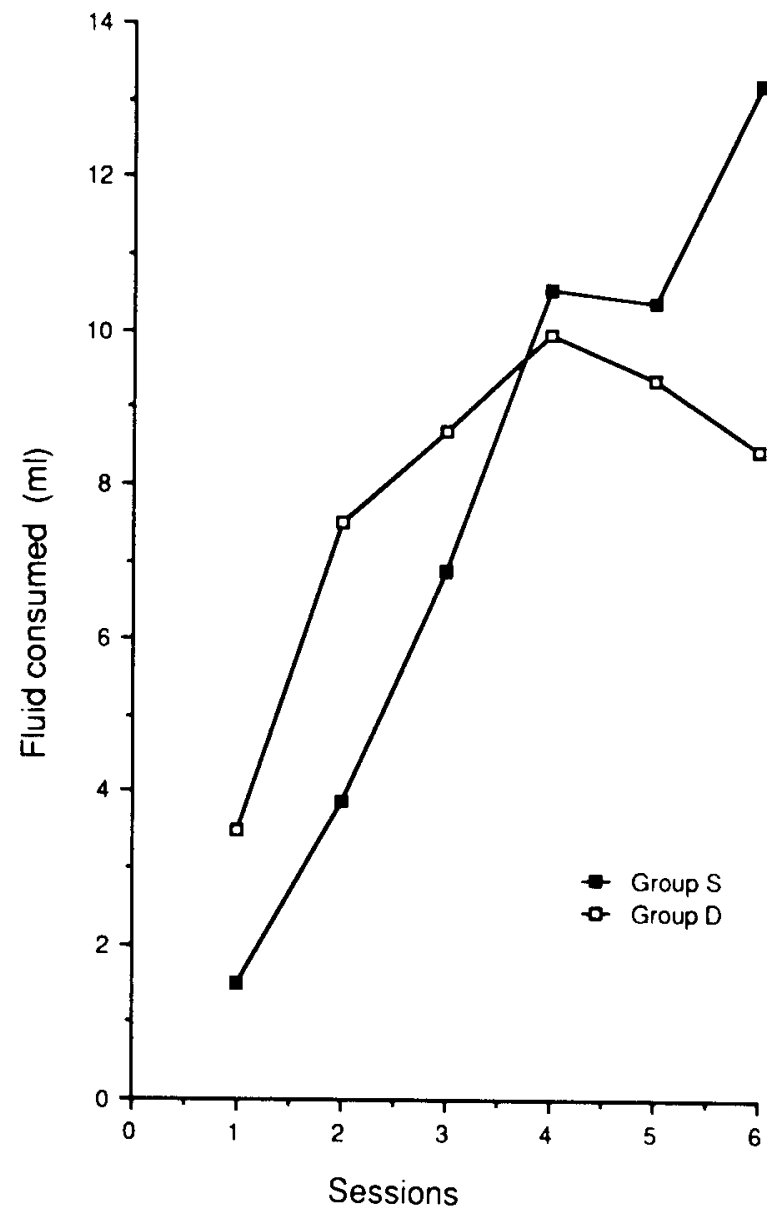

Figure 1. Group mean consumption of Flavor $T$ during the test sessions of Experiment 1.

being the consequence of the formation of a context-flavor association, but again this presumably played no role in our procedure, since our $\mathrm{S}$ and $\mathrm{D}$ groups did not differ; nor does the related idea that training context and flavor might interact to form a unique configural cue that acts as the CS to which the CR is conditioned-such a mechanism would also have produced a loss of responding in Group D. It appears that we have successfully eliminated a variety of sources of context dependency in flavor aversion, some of which are of no great theoretical interest. But equally we have failed to find the form of context specificity that we sought; we have no evidence that the context can act as a conditional cue.

\section{EXPERIMENT 2}

Without a fully specified theory of the mechanisms whereby a cue might exert conditional control over an association, it is difficult to be sure about what would be the best procedures for establishing such control. It might be argued, however, that those used in Experiment 1 were less than ideal. In particular, the subjects experienced only 
a single conditioning trial with the target flavor. It seems reasonable to assume that the chances of contextual control being established would be increased if the number of conditioning trials in that context were increased. In the present experiment, therefore, we used essentially the same design as that of Experiment 1, but five conditioning trials were given with each flavor.

\section{Method}

Subjects and Apparatus. The subjects were 16 male Wistar rats with a mean free-feeding weight of $306 \mathrm{~g}$ (range: $270-340 \mathrm{~g}$ ). The apparatus was the same as in Experiment 1.

Procedure. As in Experiment 1, a schedule of water deprivation was established, and on Day 1 of the experiment proper the subjects received access to water in each of the two contexts.

Conditioning began on the next day. The procedure on Days 2 and 3 was identical to that used in Experiment 1, except that the $\mathrm{LiCl}$ concentration was reduced to $0.1 \mathrm{M}$. This 2-day cycle was repeated five times, so that there was a total of five conditioning trials with each flavor.

Testing began on Day 12. The test procedure was identical to that used in Experiment 1.

\section{Results and Discussion}

Figure 2 shows mean fluid consumption during the conditioning trials and the test phase. As the left side of the figure shows, the conditioning trials were effective in reducing the consumption of both flavors in the two main experimental groups. There is some suggestion from the figure that the animals in both groups drank slightly more of Flavor $\mathrm{T}$ than of Flavor C, and that Group D tended to drink slightly more than Group S, but an ANOVA with group ( $\mathrm{S}$ or $\mathrm{D}$ ), flavor (T or $\mathrm{C}$ ), and sessions as factors showed that neither of these differences was significant. There was a significant main effect of sessions $[F(4,56)=$ $60.79, p<.01]$, but no other effects or interactions were significant [largest $F(1,14)=3.43$ ] .

The results of the test stage are shown on the right side of Figure 2. It is apparent that the groups differed little early in testing, but that as the aversion began to diminish, a difference emerged, with Group D showing substantially less evidence of aversion than Group $S$. An ANOVA with group (S or D), test context (A or B), and sessions as factors revealed a main effect of sessions $[F(5,60)=20.21, p<.01]$, no main effect of group $[F(1,12)=1.11]$, but a significant group $\times$ sessions interaction $[F(5,60)=2.48, p<.05]$. Interpretation of this difference is complicated by the fact that there was also a significant main effect of test context $[F(1,12)=8.76$, $p<.05]$ and a significant group $\times$ test context interaction $[F(1,12)=6.20, p<.05]$. In Context B, Group $S$ drank substantially less per session $(1.85 \mathrm{ml})$ than did Group $\mathrm{D}(9.40 \mathrm{ml})$, a difference that was statistically reliable $[t(12)=2.51]$. In Context $A$, in which the subjects drank much more, the difference between the groups (Group S, $13.46 \mathrm{ml}$; Group D, $10.40 \mathrm{ml}$ ) was not reliable $[t(12)=1.02]$. This pattern of results is perhaps most parsimoniously interpreted as suggesting that the context specificity of the aversion can be obscured (by a "ceiling effect") when levels of consumption are high.
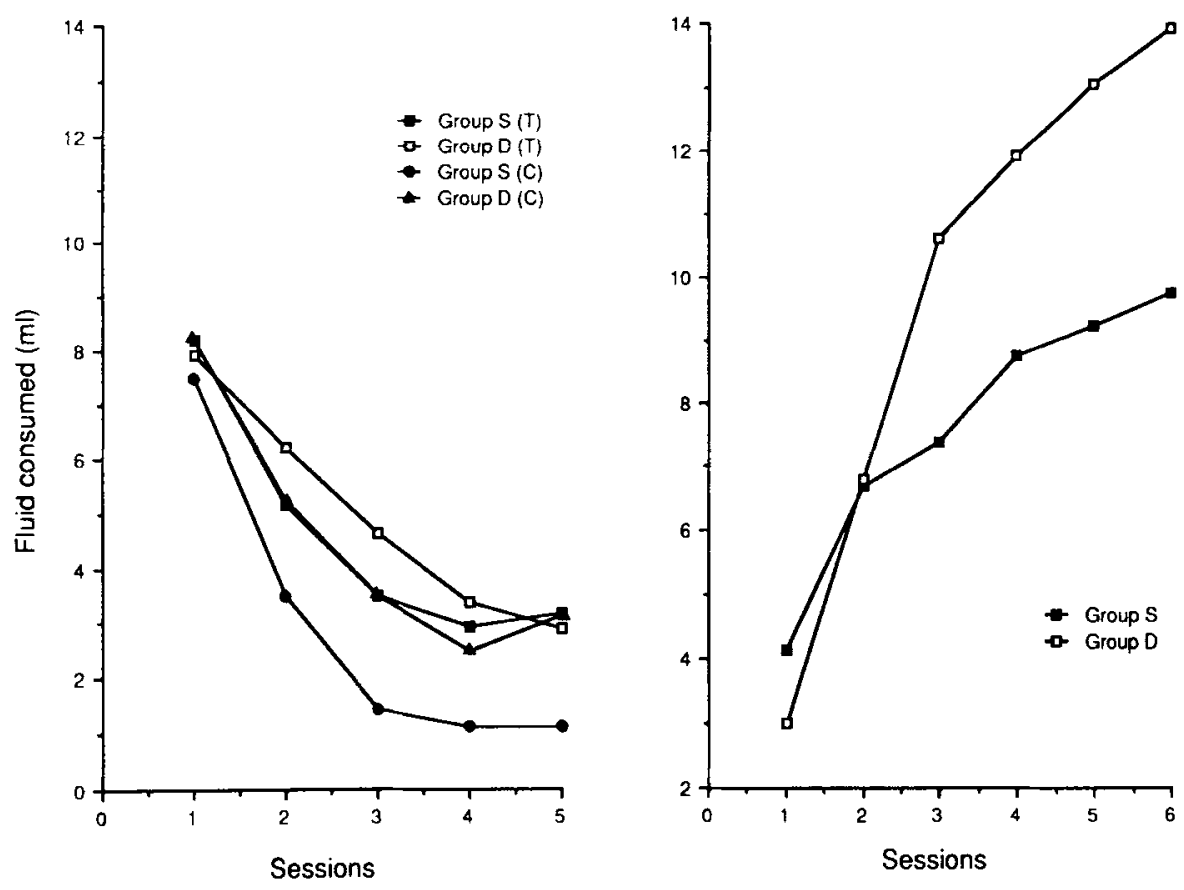

Figure 2. Mean consumption by Group $S$ and Group D of the target flavor (T) and the control flavor (C) during the conditioning phase (left panel) and test sessions (right panel) of Experiment 2. 
In the present experiment, a conditioned flavor aversion was extinguished more rapidly after a change of context. This is consistent with the possibility that the context change reduced the strength of the conditioned aversion. But no controls were included in the present experiment to rule out the possibility that this result reflects an unconditioned effect of context change on fluid consumption-even though there was no evidence for such an effect in the one-trial procedure of Experiment 1. Unpublished data from this laboratory suggest, however, that this account is incorrect. In an experiment identical to Experiment 2 except for the fact that $\mathrm{LiCl}$ injections followed context sessions during which the animals received water rather than the flavors, context change was found to have no effect on flavor consumption. Indeed, the animals tested in a different context drank nonsignificantly less than those tested in the context in which the flavor had been presented. It seems unlikely, therefore, that the present results can be attributed to an unconditioned effect of context change on fluid consumption.

Before accepting these results as showing that, given enough conditioning trials, contextual cues can gain conditional control over the CS-US association, a further interpretation should be considered. Although there has been debate about the extent to which conditioning shows context specificity, there is no doubt that latent inhibition does so. It has been found for a range of different training preparations that the retardation of conditioning produced by preexposure to the to-be-CS is attenuated when preexposure and conditioning occur in different contexts (e.g., Channell \& Hall, 1983; Hall \& Channell, 1986; Hall \& Honey, 1989; Lovibond et al., 1984). Latent inhibition of a sort can even occur when the target stimulus is accompanied by a reinforcer during the preexposure phase (Hall \& Pearce, 1979; Schachtman, Channell, \& Hall, 1987), and this form of latent inhibition too is attenuated by a change of context (Swartzentruber \& Bouton, 1986). Although there are as yet no demonstrations of these effects in flavor-aversion procedures, it is nonetheless possible to construct the following tentative account of the present results. Perhaps the extended training given to the target flavor in the present experiment resulted in latent inhibition, and, if so, further learning involving that flavor would occur only slowly provided the context remained the same. The test phase of the present experiment was one that required further learning in that an extinction procedure was used, resulting in a loss of the conditioned aversion. And, as Figure 2 shows, the two main groups differed not so much in the level of the conditioned aversion shown as in the rate at which they lost it. Perhaps the higher level of consumption shown by Group D is just a further demonstration of the context specificity of latent inhibition.

The analysis just outlined follows directly from the account of contextual effects offered by Wagner (1976, 1979,1981 ). Wagner has argued that the presentation of a CS in a given context will allow the formation of a con-
text-CS association. Presentation of the context is then able, in the terminology used by Wagner (1981), to generate a secondary state of activation (A2) in the central representation of the CS. A2 activity limits the extent to which the presentation of the stimulus itself can evoke a primary state of activation (A1), a state that is more effective at evoking a CR and is required for formation of further associations. But if the CS is presented in a novel context, it should be able to evoke A1. In consequence, it might be especially effective in eliciting its $\mathrm{CR}$, at least initially, and it should also be especially ready to engage in the processing involved in an extinction procedure that would cause a loss of the CR. It is apparent that the pattern of results presented in Figure 2 is entirely compatible with Wagner's account. Accordingly, we conducted a third experiment intended to allow discrimination between this interpretation and the suggestion that the context acts as a conditional cue.

\section{EXPERIMENT 3}

The conditional-cue account of context effects has been expressed in many different ways (see, e.g., Bouton \& Bolles, 1985; Medin, 1976; Miller \& Schachtman, 1985; Spear, 1973), but all of them incorporate some version of the notion that information about a CS-US relationship can be used or retrieved only in the presence of appropriate contextual cues. In the absence of contextual cues, the degree to which the CS predicts the US will be reduced. One consequence will be a decrement in the magnitude of the CR, but other effects can be expected as well. For example, in a blocking procedure, the degree to which an animal learns about a stimulus is reduced by the concurrent presence of a second stimulus that has already been trained as a predictor of the US. If a change of context reduces the effectiveness of the pretrained stimulus as a predictor of the US, then this stimulus should also be less effective at blocking the acquisition of associative strength by the added stimulus. The results presented in Figure 2 can be interpreted as showing that Group D is less able to activate its associate, and hence the CR, because of the contextual change. If this interpretation is correct, then we might expect that Group D's CS would also be a rather inadequate blocking stimulus.

The suggestion that contextual change has its effect by restoring the lost effectiveness or associability of a pretrained CS leads to quite different predictions. As we have already seen, Wagner's $(1976,1981)$ theory suggests that presenting a CS in a new context should lead to an enhancement in its ability to activate the US representation and elicit the CR. In Experiment 2, it can be argued that this effect was obscured by the rapidity with which further learning (extinction) occurred. But if, instead of an extinction test, the test phase was one in which the pretrained stimulus continued to be reinforced in compound with some other stimulus, then its true effectiveness as a CS should be revealed. The restored as- 
sociability of the CS would be irrelevant here, but the heightened ability of the CS to signal the US should make it an especially effective blocking stimulus.

In order to investigate these divergent predictions, we exploited the fact that there is evidence for blocking in flavor-aversion learning (e.g., Gillan \& Domjan, 1977; Revusky, 1971; see also Batson \& Best, 1979) and we conducted a blocking version of Experiment 2. The initial stage of conditioning was conducted just as in the previous experiment, but the second stage consisted of compound conditioning in which the presentation of a novel flavor (sucrose) accompanied further reinforced trials with the pretrained flavor. For half the subjects (Group S), this compound phase occurred in the same context as that used to pretrain the blocking stimulus; for the remainder (Group D), a different context was used. In a final test, all subjects received a presentation of sucrose alone in the context used for compound conditioning. Differences between the groups in consumption on the test can be taken to indicate differences in the extent to which the pretrained element had successfully blocked the acquisition of an aversion.

\section{Method}

Subjects and Apparatus. The subjects were 16 male Wistar rats with a mean free-feeding weight of $389 \mathrm{~g}$ (range: $350-440 \mathrm{~g}$ ). They were housed in pairs, as in Experiment 2. The apparatus was identical to that used in Experiment 2.

Procedure. The water-deprivation schedule and preliminary sessions of exposure to water in the contexts were identical to those in Experiment 2. Conditioning began on Day 2. A 2-day cycle identical to that used in Experiment 2 was used. This cycle was repeated four times, so that a total of four conditioning trials to each of the flavors was administered.
There followed six sessions of compound training, for which the animals were divided into two groups exactly as in Experiment 1. For Group S, compound trials occurred in the context in which the target flavor had been conditioned; for Group D, compound trials occurred in the other context.

Compound trials were administered in the following way. The subjects were placed in the context for $15 \mathrm{~min}$, after which they received access to $5 \mathrm{ml}$ of Flavor $\mathrm{T}$ for $15 \mathrm{~min}$. Immediately after this presentation they received access to $5 \mathrm{ml}$ of $0.33 \mathrm{M}$ sucrose solution for an additional $15 \mathrm{~min}$. After $1 \mathrm{~h}$ they were returned to the home cage, from which they were removed and given a $10-\mathrm{ml} / \mathrm{kg}$ injection of $0.1 \mathrm{M} \mathrm{LiCl}$. The subjects continued to experience two context sessions per day. In the session in the other context, they received two consecutive 15 -min presentations of $5 \mathrm{ml}$ of water.

Test trials occurred in the context in which the compound trials had occurred. The animals remained in the test context for $15 \mathrm{~min}$ and then received free access to sucrose solution for $15 \mathrm{~min}$. During their other daily session, they received water exactly as in the compound conditioning stage. This test stage lasted for 4 days.

\section{Results and Discussion}

Figure 3 shows mean fluid consumption during the conditioning trials and the test phase. During the initial flavorconditioning trials, both groups readily acquired aversions to both flavors, as the left panel of the figure shows. An ANOVA with group (S or D), flavor (T or C), and sessions as factors revealed only a significant main effect of sessions $[F(3,42)=65.05, p<.01]$. There were no other significant effects or interactions $(F \mathrm{~s}<1)$.

Context specificity of conditioning might conceivably have been observed in the compound-conditioning phase when Group D first experienced Flavor $T$ in a different context. But, as the middle panel of Figure 3 shows, all animals continued to consume only small amounts of
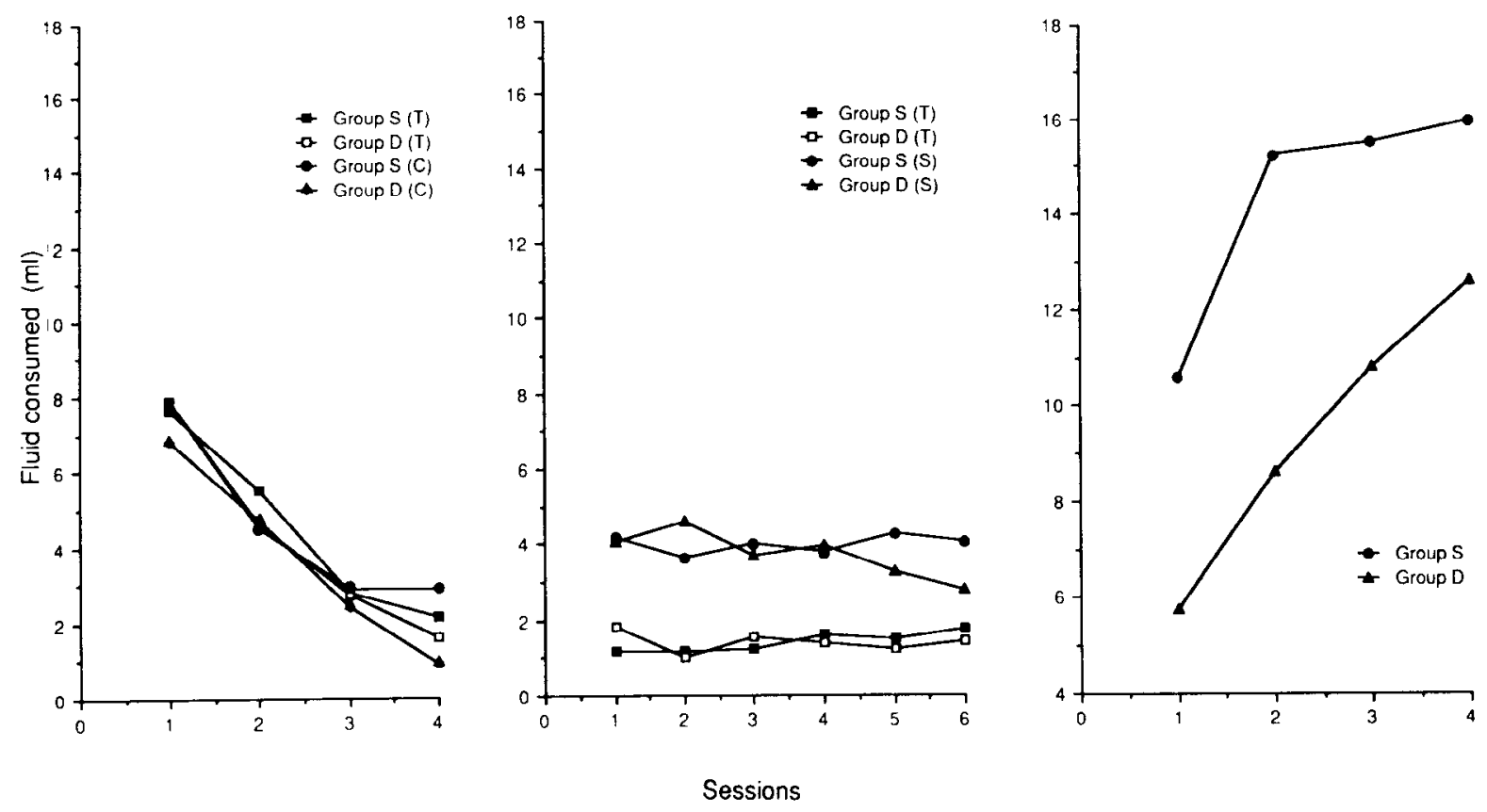

Figure 3. Mean consumption by Group $S$ and Group D of the target flavor (T), the control flavor (C), and sucrose (S) during element conditioning (left panel), compound conditioning (middle panel), and the test phase (right panel) of Experiment 3. 
Flavor $\mathrm{T}$, and any difference between the two groups might have been obscured by a "floor effect." An ANOVA with group (S or D) and sessions as factors revealed no significant effects or interactions [largest $F(5,70)=1.51]$. Although the scope for seeing the development of an aversion to sucrose was limited by the fact that only $5 \mathrm{ml}$ of the solution was made available on each trial, there was some sign that an aversion had developed in Group D by the final two trials of this stage. An ANOVA with group ( $S$ or $D$ ) and sessions as factors revealed a significant group $\times$ sessions interaction $[F(5,70)=3.82, p<.01]$. No other effects or interactions were significant [largest $F(5,70)=1.47]$. The more rapid development of an aversion to sucrose in Group D is what might be expected if the change of context reduced the ability of Flavor $T$ to act as a blocking stimulus.

The results of the test stage are shown in the right panel of Figure 3, and they confirm that blocking was less effective in Group D. Group S drank more sucrose than Group D throughout the test phase. An ANOVA with group (S or D), test context (A or B), and sessions as factors revealed a significant main effect of group $[F(1,12)=$ $7.74, p<.05]$. There was also a significant main effect of sessions $[F(3,36)=10.20, p<.01]$ and a main effect of test context $[F(1,12)=12.21, p<.01]$. The effect of test context was a consequence of the fact that, on average, more fluid was drunk during sessions in Context A $(14.97 \mathrm{ml})$ than in Context B $(8.83 \mathrm{ml})$. No other effects or interactions were significant [largest $F(1,12)=$ 3.72].

We conclude, on the basis of these results, that a CS's ability to block the acquisition of associative strength by a second stimulus is attenuated by a change of context. Such a conclusion should, however, be qualified by the observation that no controls for blocking were included in the present experiment. It may be pointed out, however, that the procedures used here were similar to those employed in previous studies by Gillan and Domjan (1977) in which blocking has been successfully demonstrated. It remains possible, in principle, that blocking was not obtained with our parameters, but if this were the case, an alternative interpretation would be required for our results, and it is not at all clear what form such an alternative interpretation might take.

If these data may be interpreted as evidence for attenuation of blocking with a change of context, such a result is not to be expected on the basis of the suggestion (e.g., by Wagner, 1976, 1979, 1981) that a CS is rendered more effective (both more associable and more potent at activating its associate) by a change of context. It accords very well, however, with the notion that use of the information embodied in a CS-US association can be conditional upon the presence of appropriate contextual cues.

\section{GENERAL DISCUSSION}

In Experiment 1, rats given a single conditioning trial with a flavor CS drank as much of that flavor when they were tested in the conditioning context as when they were tested in another context in which a different flavor had been trained. This suggests that the aversion that was acquired transferred perfectly to a different context. But context specificity was evident in two further experiments. In Experiment 2, which differed from Experiment 1 only in that more conditioning trials were administered, the subjects showed more rapid extinction of a conditioned aversion after a change of context. In Experiment 3, sucrose was conditioned in compound with a pretrained flavor. Subsequent sucrose consumption was greater if compound conditioning took place in the context in which the pretrained flavor had been conditioned than if it took place in another context in which a different flavor had been conditioned. This suggests that presenting the pretrained stimulus in a new context reduced its ability to block the acquisition of associative strength by the sucrose. This last finding would not be expected if the sole effect of the change of context was to restore the associability that the pretrained stimulus might have lost during the first stage of training. Rather, it seems that when a flavor occurs outside the accustomed training context, that flavor is less capable of acting as a CS (i.e., of activating its associate and eliciting the $\mathrm{CR}$ ).

There are several possible reasons why an aversion conditioned to a flavor in one context might fail to appear when the flavor is presented in some other context. The new context might modify the way in which the flavor is perceived, producing generalization decrement; associations between the context and the reinforcer that contribute to the aversion observed during conditioning might not contribute during testing; or contextual cues might play a part in enabling the retrieval or use of the information embodied in a flavor-US association. We tend toward the conclusion that the context specificity demonstrated in Experiments 2 and 3 reflects the operation of the last of these mechanisms. Our reasons include the following. First, we chose stimuli and procedures likely to minimize the role of generalization decrement; second, we arranged that the test context should have the same associative strength as the training context; and third, no evidence of context specificity was found in Experiment 1, in spite of the fact that the same stimuli and general training procedures were used, and thus the chances of generalization decrement occurring might be thought to be much the same as in Experiment 2. The special feature of Experiment 2 was that more extensive reinforced training was given. We argued that the single reinforced trial used in Experiment 1 might not have been sufficient to establish the context as a conditional cue controlling the CS-US association, but that such control would be acquired if the subjects were given more training.

We should acknowledge, however, that these arguments do not allow us to rule out a further possible source of context specificity. A conditioned response would fail to transfer to a new context if the effective CS were some configure-that is, some unique cue-created by the interaction of the nominal CS and contextual cues. Since 
it has been argued that configural cues will form spontaneously (i.e., without explicit discrimination training) only when prolonged training is given (Bellingham \& Gillette, 1981), this interpretation leads to the expectation that context specificity will be unlikely with just one conditioning trial. As with several of the other procedures that have been used to investigate conditional learning (Holland, 1983), there is little in the data that allows the choice between the configural-cue account and its rival. However, if the configural account were correct, presenting the flavor in a different context would alter the nature of the configure so that one would expect a return of neophobia with a change of context. We have unpublished data that demonstrate, however, that there is no evidence for such an effect with our experimental procedures. Preference for the notion that the context functions as a separate cue controlling the CS-US association comes, moreover, from the fact that this interpretation is better able to accommodate the results of related experimental work reported by Hall and Honey (1989).

If our conclusion is justified, then the experiments presented here constitute further evidence that contextual cues can gain conditional control over the CS-US association even without explicit discrimination training, in a simple conditioning procedure-that is, without training in which the CS is presented in some other context but is not followed by the US. Moreover, such conditional control can be found despite the use of an aversive reinforcer. This suggests that the occurrence of context specificity in simple conditioning is not confined to appetitive procedures, as its absence in conditioned suppression (Hall \& Honey, 1989); Lovibond et al., 1984) might be taken to suggest. Hall and Honey suggest that the difference between appetitive and aversive preparations could be a result of the aversive reinforcer disrupting discrimination of the conditioned stimuli. The present results could be taken as consistent with this possibility, if one is prepared to concede that such disruption would be less probable when the delay between the CS and the US is long, as is the case in a flavor-aversion procedure. In the absence of further experimental evidence that might lend weight to such speculation, however, we are left with the conclusion that, in some circumstances, context specificity may be found with simple aversive conditioning procedures.

\section{REFERENCES}

ARCher, T. , \& SuödÉN, P.-O. (1980). Context-dependent taste-aversion learning with a familiar conditioning context. Physiological Psychology, 8, 40-46.

ArCher, T., SuödÉn, P.-O., \& Nilsson, L.-G. (1985). Contextual control of taste-aversion conditioning and extinction. In P. D. Balsam \& A. Tomie (Eds.), Context and learning (pp. 225-271). Hillsdale, NJ: Erlbaum.

Archer, T., SJödén, P.-O., Nilsson, L.-G., \& Carter, N. (1979). Role of exteroceptive background context in taste-aversion conditioning and extinction. Animal Learning \& Behavior, 7, 17-22.

archer, T., Suödén, P.-O., Nilsson, L.-G., Carter, N. (1980).
Exteroceptive context in taste-aversion conditioning and extinction: Odor, cage, and bottle stimuli. Quarterly Joumal of Experimental Psychology, 32, 197-214.

Batson, J. D., \& Best, P. J. (1979). Drug-preexposure effects in flavoraversion learning: Associative interference effects by conditioned environmental stimuli. Joumal of Experimental Psychology: Animal Behavior Processes, 5, 273-283.

Bellingham, W. P., \& Gillette, K. (1981). Spontaneous configuring to a tone-light compound using appetitive training. Learning \& Motivation, 12, 420-434.

Bouton, M. E. (1988). Context and ambiguity in the extinction of emotional learning: Implications for exposure therapy. Behaviour Research \& Therapy, 26, 137-149.

Bouton, M. E., \& Bolles, R. C. (1985). Contexts, event memories, and extinction. In P. D. Balsam \& A. Tomie (Eds.), Context and learning (pp. 133-166). Hillsdale, NJ: Erlbaum.

Bouton, M. E., SWARTzentruber, D. (1986). Analysis of the associative and occasion-setting properties of contexts participating in a Pavlovian discrimination. Journal of Experimental Psychology: Animal Behavior Processes, 12, 333-350.

Channell, S., \& Hall, G. (1983). Contextual effects in latent inhibition with an appetitive conditioning procedure. Animal Learning \& Behavior, 11, 67-74.

Gillan, D. J., \&omjan, M. (1977). Taste-aversion conditioning with expected versus unexpected drug treatment. Joumal of Experimental Psychology: Animal Behavior Processes, 3, 297-309.

Hall, G., Channell, S. (1986). Context specificity of latent inhibition in taste-aversion learning. Quarterly Journal of Experimental Psychology, 38B, 121-139.

HALL, G., HoneY, R. C. (1989). Contextual effects in conditioning, latent inhibition, and habituation: Associative and retrieval functions of contextual cues. Journal of Experimental Psychology: Animal Behavior Processes, 15, 232-241

Hall, G. Pearce, J. M. (1979). Latent inhibition of a CS during CS-UCS pairings. Journal of Experimental Psychology: Animal Behavior Processes, 5, 31-42.

Holder, M. D. (1988). Possible role of confounded taste stimuli in conditioned taste aversions. Animal Learning \& Behavior, 16, 231-234.

Holland, P. C. (1983). "Occasion setting" in Pavlovian feature positive discriminations. In M. L. Commons, R. J. Hermstein, \& A. R. Wagner (Eds.), Quantitative analyses of behavior: Discrimination processes (Vol. 4, pp. 183-206). New York: Ballinger.

Lovibond, P. F., Preston, G. C., \& Mackintosh, N. J. (1984). Context specificity of conditioning and latent inhibition. Journal of Experimental Psychology: Animal Behavior Processes, 10, 360-375.

MEdin, D. L. (1976). Animal models and memory models. In D. L. Medin, W. A. Roberts, \& R. T. Davis (Eds.), Processes of animal memory (pp. 113-134). Hillsdale, NJ: Erlbaum.

Miller, R. R., \& Schachtman, T. R. (1985). The several roles of context at the time of retrieval. In P. D. Balsam \& A. Tomie (Eds.), Context and learning (pp. 167-194). Hillsdale, NJ: Erlbaum.

Preston, G. C., Dickinson, A., \& Mackintosh, N. J. (1986). Contextual conditional discriminations. Quarterly Journal of Experimental Psychology, 38B, 217-237.

REVUSKY, S. (1971). The role of interference in association over a delay. In W. K. Honig \& P. H. R. James (Eds.), Animal memory (pp. 95-128). New York: Academic Press.

Schachtman, T. R., Channell, S., Hall, G. (1987). Effects of CS preexposure on inhibition of delay. Animal Learning \& Behavior, $15,301-311$

SJÖDÉN, P.-O., \& ARChER, T. (1988). Exteroceptive cues in tasteaversion learning, no artifact: Reply to Holder. Animal Learning \& Behavior, 16, 235-239.

SPEAR, N. E. (1973). Retrieval of memory in animals. Psychological Review, 80, 163-194.

Swartzentruber, D., \& Bouton, M. E. (1986). Contextual control of negative transfer produced by prior CS-US pairings. Learning \& Motivation, 17, 366-385.

WAGNER, A. R. (1976). Priming in STM: An information-processing mechanism for self-generated or retrieval-generated depression in per- 
formance. In T. J. Tighe \& R. N. Leaton (Eds.), Habituation: Perspectives from child development, animal behavior, and neurophysiology (pp. 95-128). Hillsdale, NJ: Erlbaum.

Wagner A. R. (1979). Habituation and memory. In A. Dickinson \& R. A. Boakes (Eds.), Mechanisms of learning and motivation (pp. 53 82). Hillsdale, NJ: Erlbaum.

WAGNER, A. R. (1981). SOP: A model of automatic memory process- ing in animal behavior. In N. E. Spear \& R. R. Miller (Eds.), Information processing in animals: Memory mechanisms (pp. 547). Hills dale, NJ: Erlbaum

(Manuscript received March 1, 1989;

revision accepted for publication August 30, 1989.) 